\title{
Streptococcus mitis
}

National Cancer Institute

\section{Source}

National Cancer Institute. Streptococcus mitis. NCI Thesaurus. Code C89774.

A mesophilic, alpha-hemolytic species of Streptococcus that is a normal constituent of human mouth flora but that can cause endocarditis. 